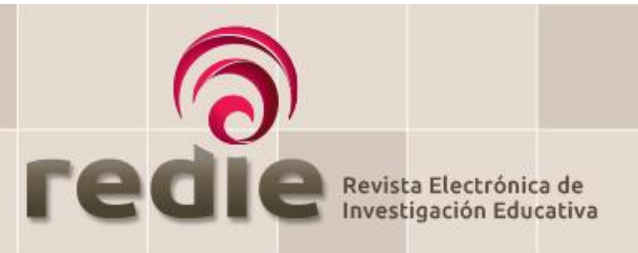

Vol. 19, Núm. 4, 2017

\title{
Un Men's Shed como comunidad favorecedora del bienestar en adultos mayores
}

\section{A Men's Shed as Community that Facilitates Well-Being in the Elderly}

\author{
Alicia González Pérez (*) aliciagp@unex.es \\ Manuel Rafael de Besa Gutiérrez (*)mandegut@alum.us.es \\ (*) Universidad de Extremadura \\ (Recibido: 1 de abril de 2016; Aceptado para su publicación: 6 de junio de 2016)
}

Cómo citar: González, A. y De Besa, M. R. (2017). Un Men's Shed como comunidad favorecedora del bienestar en adultos mayores. Revista Electrónica de Investigación Educativa, 19(4), 134-144. https://doi.ora/10.24320/redie.2017.19.4.1336

\section{Resumen}

Los Men's Shed se caracterizan por ser un tipo de comunidad formada fundamentalmente por hombres de edad avanzada y en riesgo de exclusión social. Este artículo es parte de una investigación europea que se desarrolla en Faro (Portugal) y cuyo objetivo es analizar las implicaciones de los Men's Shed en el bienestar y la salud, aprendizaje informal, socialización y sentido de pertenencia. La muestra del estudio está integrada por los 5 miembros del Club de pesca "Los amadores", de Faro. Para recoger los datos se aplicó la técnica de entrevista narrativa; posteriormente se analizaron las entrevistas a través del programa de análisis cualitativo Atlas.ti v5. Los resultados obtenidos proporcionan evidencias claras de que este tipo de comunidades favorecen y propician el bienestar de sus integrantes, así como el sentido de pertenencia a través del desarrollo de aprendizajes informales.

Palabras clave: Aprendizaje informal, Men's Shed, Centros comunitarios, Salud y bienestar.

\section{Abstract}

Men's sheds are characterized as a type of community essentially made up of older men at risk of social exclusion. This paper is part of a European study being performed in Faro, Portugal, the aim of which is to analyze the impact of men's sheds on health and well-being, informal learning, socialization and sense of belonging. The study sample is made up of 5 members of the "Los Amadores" fishing club in Faro. Narrative interviews were used to collect data, and the interviews were then analyzed using the qualitative analysis program Atlas.ti v5. The results obtained offer clear evidence that this type of community facilitates and promotes well-being in members, as well as a sense of belonging through informal learning. 


\section{Introducción}

La jubilación es una etapa de la vida en la que los mayores pueden experimentar una pérdida de identidad (Ballinger, Talbot y Verrinder, 2009). Mientras que algunos estudios abordan las dificultades que los mayores afrontan al intentar remplazar las rutinas y demandas del trabajo, son menos los estudios que se centran en cómo gestionar el cambio al pasar de ser trabajador en activo a jubilado (Ballinger et al., 2009). Es por ello que en los últimos años ha crecido el movimiento Men's Shed, especialmente en Australia (Golding et al., 2008; Ballinguer et al., 2009). En la revisión sobre la literatura acerca de los Men's Shed, Wilson y Cordier (2013) concluyen que la mayoría de los estudios provienen de Australia y se centran en el aprendizaje de hombres en edad avanzada en contextos comunitarios.

Según Golding, Foley y Brown (2007) los Men's Shed nacieron a mediados de los noventa y se definen de manera muy amplia, como "un tipo de espacios de convivencia en una comunidad donde se desarrollan talleres regulares y actividades manuales por grupos principalmente de varones". Están dirigidos a adultos mayores que suelen compartir características similares y la mayoría tiene que abordar situaciones como la soledad, la jubilación, el desempleo, enfermedades físicas o mentales u otras situaciones personales. Lo peculiar de estos grupos es que en ellos se produce un aprendizaje informal a través del desarrollo de actividades y de las relaciones entre sus componentes. Además, también pueden darse relaciones intergeneracionales en las que los conocimientos y aprendizajes pasan de los más viejos a los más jóvenes, por ello es importante conocer las implicaciones que tienen los Men's Shed en cuestiones como el bienestar y la salud, el aprendizaje informal, la socialización y el sentido de pertenencia.

\subsection{Implicaciones de los Men’s Shed en la mejora de la salud y el bienestar de los mayores}

La salud y el bienestar de los hombres es una cuestión que ha ido ganando la atención de investigadores, responsables políticos y profesionales de la salud en las dos últimas décadas. Diferencias entre las tasas de mortalidad de género indican que en los países occidentales los hombres y niños experimentan un deterioro de su salud mientras que las mujeres y niñas experimentan bienestar (White y Holmes, 2006). De acuerdo con Wilson y Cordier (2013) existen grupos de mayores que verdaderamente están en riesgo de exclusión debido, fundamentalmente, a que viven en zonas rurales o áreas remotas, son hombres con enfermedades crónicas, desempleados, con una educación limitada, pertenecen a minorías culturales o a una escala social baja.

De ahí que Misan, Haren y Ledo (2008) sugieren que Men's Shed tienen el potencial de "lograr resultados positivos en salud, felicidad y bienestar de los hombres que participan, así como de sus parejas, familiares y comunidades". A través de los estudios de Labonte (1997) y Ballinger et al. (2009) se han identificado seis elementos que repercuten positivamente en la salud de las personas mayores, estos son: el sentirse lleno de vida y energía, tener buenas relaciones sociales, experimentar la sensación de control sobre la propia vida, sentirse capaz de hacer algo con lo que puede disfrutar, tener un propósito en la vida y experimentar una conexión con la comunidad.

Ballinger et al. (2009) consideraron muy significativa la participación de los mayores en los Men's Shed financiados por las autoridades locales. Además, observaron cómo éstos podían impactar la salud y el bienestar de los hombres de forma positiva. Sus hallazgos sugirieron que los Men's Shed son un lugar significativo para congregarse, que sirven para mejorar el bienestar de los hombres, en general, y el de los que tienen algún problema de salud mental o son adictos a sustancias, en particular. Por ello, los Men's Shed han de ser para los responsables políticos una parte importante de la amplia gama de respuestas que tiene la política para mejorar la salud y el bienestar de los hombres (Wilson y Cordier, 2013), ya que, alivian el aislamiento social y mejoran el bienestar de los mayores. Ejemplos de políticas en este sentido se dan en Australia e Irlanda (Robertson, Galdas, McCreary, Oliffe y Tremblay, 2009; Smith, White, Richardson, Robertson y Ward, 2009). 


\subsection{Aprendizaje informar de varones adultos en asociaciones de voluntariado}

El aprendizaje y la educación informal son otros de los puntos fuertes de los Men's Shed debido al potencial de aprendizaje que tienen este tipo de instituciones para los mayores. Golding y su equipo han sido los que han dirigido más investigaciones acerca del aprendizaje de adultos en el contexto de los Men's Shed (Golding, 2011a; 2011b; Golding, Kimberly, Foley y Brown, 2008; Golding, Foley y Brown, 2007). En estos estudios se afirmaba que los Men's Shed ofrecen un contexto de aprendizaje informal que se adapta mejor a los hombres que los tradicionales centros de aprendizaje. Esto significa que los mayores no están interesados en contextos de aprendizaje formal, ya que su deseo es desarrollar nuevas habilidades y estos contextos informales facilitan ese tipo de aprendizajes (Wilson y Cordier, 2013). Por tanto, son lugares idóneos para conectar con otros miembros, para aprender habilidades prácticas y desarrollar nuevos intereses.

Golding et al. (2008) asocian el aprendizaje con el bienestar; sin embargo, esta asociación no está probada y, por tanto, no puede ser corroborada. A pesar de esta limitación, los trabajos de Golding sugieren que los Men's Shed facilitan más la participación de adultos en contextos de aprendizaje informal que los tradicionales centros de aprendizaje. Esto es, los adultos no están interesados en ambientes de aprendizaje formal, pero el deseo de desarrollar nuevas habilidades y los Men's Shed como contextos informales facilitan este tipo de aprendizajes.

En una de sus investigaciones, Golding et al. (2009) se centraron específicamente en dos puntos clave: los aprendizajes de personas mayores y el bienestar a través de la participación en seis tipos de comunidades (proveedores de educación comunitaria y de adultos, organizaciones que prestan especial atención a los hombres, voluntariado de servicios de bomberos y emergencias, clubes deportivos, organizaciones religiosas, indígenas y culturales, y otras).

En una muestra de 219 hombres adultos pertenecientes a las comunidades especificadas, el aprendizaje fue destacado como algo muy favorable. Por ejemplo, el $94 \%$ de la muestra estaba de acuerdo en su disposición a aprender más, un $95 \%$ estaba dispuesto a mejorar sus habilidades y un $91 \%$ consideró que formar parte de la organización les ayudó a aprender. Cerca de la mitad (48\%) estaba de acuerdo en que la organización debería de ofrecer más oportunidades para aprender. Mientras que sus preferencias eran aprendizajes prácticos (72\%), en la organización (76\%), la mayoría de ellos estaba de acuerdo en que preferían aprender en situaciones prácticas (95\%) y haciendo (97\%) (Golding, 2011a).

En resumen, y según la literatura revisada, la actividad regular, social y práctica -por ejemplo la jardinería, la cerámica, la carpintería y la pesca- son actividades especialmente valoradas por los mayores en contextos de aprendizaje informal. Fundamentalmente predominan hombres mayores con un alto nivel de compromiso. Sin embargo, dada la importancia que los Men's Shed ofrecen en términos de reducción de la exclusión social y la promoción del bienestar a través del aprendizaje, puede ser particularmente interesante extender este tipo de organizaciones a colectivos de jóvenes con dificultades de aprendizaje y de conducta.

\subsection{Socialización y la participación de mayores en comunidades de aprendizaje}

El aprendizaje y la educación pueden tener una influencia importante a la hora de reforzar las redes sociales que tienen los mayores, ya que les ofrecen distintos tipos de apoyo social que les puede ayudar a disminuir los signos de exclusión (Jelenc-Krašovec y Kump, 2009), y mejorar el bienestar en comunidad (Field 2009; Golding, 2011a).

Según algunas investigaciones desarrolladas en diferentes países, se demuestra que la proporción de personas de edad avanzada que participan en aprendizajes planificados es muy limitada. Quienes participan generalmente tienen un nivel alto de educación, son pudientes, suelen ser más activos en otras esferas de la vida y son generalmente mujeres (Formosa, 2012). Además, este tipo de instituciones varía su oferta dependiendo del tipo de población que atiende (rural o urbana), y toman en cuenta también las necesidades, expectativas, habilidades, hábitos y nivel educativo que tienen las personas mayores, entre otras. 
En consonancia con lo anterior, algunas investigaciones sobre la educación de los mayores en comunidad muestran que los hombres, especialmente los menos educados y socialmente desfavorecidos, rara vez participan de forma activa en asociaciones, además de que se excluyen de las actividades educativas. Investigaciones realizadas en Australia (Golding, 2011a; Golding et al., 2008), Canadá (Robertson, Galdas, McCreary, Oliffe y Tremblay, 2009) y Europa (McGivney, 2004) sobre la implicación de varones adultos en actividades educativas y sociales comunitarias, demuestran que los varones adultos demandan una oferta diferente para la inclusión activa en la comunidad.

Mientras que la falta de participación en actividades tiene un impacto negativo en el disfrute de la vida, la participación en actividades de ocio tiene gran importancia en el envejecimiento activo, en el disfrute de la jubilación y en la moderación de los efectos estresantes de la vida (Fitzpatrick, Spiro, Kressin, Greene y Bosse, 2001).

Los Men's Shed, además de proporcionar actividades interesantes y significativas, tienen como objetivo reforzar las interacciones sociales entre hombres, porque muchos de los que asisten pueden estar en riesgo de aislamiento social. Además, la relación entre una buena red social y la salud es algo ya establecido. Hay evidencias de la correlación entre las personas que tienen pobres redes sociales (lazos sociales débiles, desconexión social, integración social, actividad social, desarraigo social) y mortalidad. Algunos estudios han demostrado de forma consistente que las personas que están más aisladas socialmente o desconectadas de otros tienen entre dos y cinco veces más riesgo de morir en comparación con aquellos que mantienen vínculos fuertes con la familia, amigos y la comunidad (Ballinger et al., 2009).

\subsection{Identidad y sentido de la pertenencia}

Los Men's Shed son lugares amigables para hombres que tratan de contrarrestar el aislamiento social al que están expuestos y desarrollar en ellos el sentido de pertenencia a un grupo o comunidad con intereses comunes. Por ello, Golding (2011a; 2011b) y Golding et al. (2008; 2007) señalan que estos espacios proporcionan una ubicación social única para mayores varones, a la cual pueden pertenecer una vez jubilados. Además, en ellos se puede entrar en contacto con otros miembros de la comunidad mientras de forma simultánea se ofrecen oportunidades de aprendizaje y se desarrollan nuevos intereses y habilidades.

Earle, Earle y Mering (1996) encontraron que los Men's Shed ocupaban un lugar importante para la clase trabajadora masculina, y especialmente para quienes ya no pertenecían a la clase trabajadora. Estos espacios les proporcionaban un lugar donde realizar actividades aceptadas y respetadas que les ayuda a reforzar su identidad, facilita el aprendizaje y les ayuda a ser creativos; sin embargo, identificaron una limitación en su estudio en el sentido de que estos espacios privados eran más centros de aislamiento que centros sociales.

Las investigaciones de Golding et al. $(2007 ;$ 2008; 2009) identificaron uniformidad en los objetivos comunitarios basados en los Men's Shed. Para ellos, los Men's Shed en contextos comunitarios servían para compartir un compromiso común de amistad, salud y bienestar. Por ello, los Men's Shed se pueden considerar como espacios para el beneficio individual y comunitario, donde se da una práctica regular de forma frecuente y en grupo.

La necesidad del hombre de construir su propia identidad una vez que abandona el trabajo es un factor necesario para buscar actividades satisfactorias que le permitan seguir trabajando, pero sin las restricciones y limitaciones de tener un empleo. Así pues, los Men's Shed sirven a este propósito y contribuyen a mejorar su integridad, autoestima y sentido de pertenencia.

Cabe añadir que para la mayoría de los hombres los Men's Shed son algo más que un lugar para ir y hacer cosas. Son un lugar vital y en expansión que proporciona a los participantes un sentido de pertenencia y conexión con otros. 


\section{Método}

Los objetivos fundamentales de la investigación fueron: 1) Identificar los factores implicados en la mejora del bienestar y la salud de los mayores a través del aprendizaje informal, y en concreto a través del sentimiento de pertenencia al club de pesca Los Amadores; 2 ) Analizar las relaciones entre el aprendizaje informal y el bienestar y salud de las personas mayores (+60 años) dentro una comunidad de práctica, un club de pescadores, que tiene las características de los Men's Shed australianos, y 3) Analizar las relaciones sociales e intergeneracionales que se producen entre personas de diferentes edades dentro del club a través de las diferentes actividades que se planifican en el club.

El estudio se llevó a cabo en el club de pesca Los Amadores, ubicado en la capital portuguesa del Algarve (Faro), al sur de Portugal. Se caracteriza por organizar actividades sociocomunitarias para sus miembros, como son: campeonatos internos de pesca, donde los socios pueden competir entre ellos para ganarse un lugar dentro del equipo profesional, y otros campeonatos donde compiten a nivel profesional. El club está formado por 151 socios de los cuales $85 \%$ son varones frente y $15 \%$ son mujeres que acompañan a sus maridos durante las actividades, pero no son socias. De los miembros, el $52 \%$ de la muestra lo integran personas entre 40 y 64 años, y un $29 \%$ de personas jubiladas y en edad avanzada.

La mayoría de los socios se encuentran jubilados o desempleados, en situaciones desfavorables, solos sentimentalmente o con malas experiencias/vivencias. Por ello, el hecho de acudir a este club, relacionarse e interactuar con otras personas o participar en las actividades que se programan es una manera de combatir esos problemas de soledad.

Para este trabajo se optó por un enfoque metodológico cualitativo a través de un estudio de caso (Patton, 2002; Stake, 2010), que permite comprender los factores de éxito que hacen que perviva una comunidad de práctica a lo largo del tiempo donde se desarrollan aprendizajes informales. Se utiliza este enfoque por considerarlo más apropiado para investigar la complejidad de los fenómenos en un entorno natural (Creswell, 1998).

La recogida de datos se realizó a través de la técnica de entrevista narrativa semiestructurada. Primero se diseñó una batería de preguntas abiertas sobre el objeto de estudio y fue sometida a evaluación por tres expertos en el tema. Tras los análisis realizados se definieron siete categorías generales que se extrajeron del análisis realizado por los expertos y de la revisión bibliográfica. Las entrevistas se planificaron para que tuvieran una duración de 30 a 40 minutos. El objetivo егa conocer en profundidad los factores asociados a la mejora del bienestar de las personas adultas cuando son miembros de una comunidad de práctica, en este caso el club de pesca Los Amadores, donde aprenden sobre pesca y el mar de manera informal.

El diseño de las entrevista se compuso de preguntas abiertas que se centraron en las siguientes dimensiones y factores: la inserción y trayectoria en el club, actividades desarrolladas dentro del club, las relaciones sociales y conexión con la comunidad, la implicación de la familia, los aprendizaje informales que se desarrollan y la mejora de la salud y bienestar social. Por ello, se considera que el instrumento es válido en cuanto al contenido y criterio debido a que mide el contenido que pretende medir, presta especial atención a los objetivos propuestos en la investigación, y lo hace con la misma técnica de recogida de datos.

Las entrevistas fueron grabadas y transcritas para su análisis. Para la muestra se seleccionaron cinco casos que de forma voluntaria accedierona a las entrevistas. Todos ellos tenían una participación activa dentro del Club.

Para el análisis de las entrevistas se utilizó el software informático Atlas.ti v5, para análisis de datos cualitativos. Primero se codificaron todas las entrevistas narrativas identificándose unidades de significado, para luego establecer relaciones entre ellos. Por último se utilizó el programa CMaps Tools para la creación de un mapa conceptual que diera una visión general de la complejidad terminológica y sus interacciones. 
La siguiente tabla especifica las dimensiones y códigos utilizados para codificar las entrevistas narrativas.

Tabla I. Códigos utilizados en el análisis de las entrevistas narrativas

\begin{tabular}{l|l}
\hline Dimensión & Código \\
\hline Bienestar y Salud & BIEN \\
Aprendizaje informal & APRINF \\
Sentido de Pertenencia & SENTPERT \\
Socialización Intergeneracional & SOCINTER \\
Convivencia & CONV \\
Soledad & SOLED \\
Conexión con la comunidad & CON \\
\hline
\end{tabular}

\section{Resultados}

En este apartado se presentan los resultados con relación a los objetivos identificados. Para ello, se elaboró el mapa conceptual que refleja la complejidad de las relaciones establecidas entre los conceptos objeto de estudio: aprendizaje informal, bienestar y salud, socialización, sentimiento de pertenencia, aislamiento social y habilidades desarrolladas.

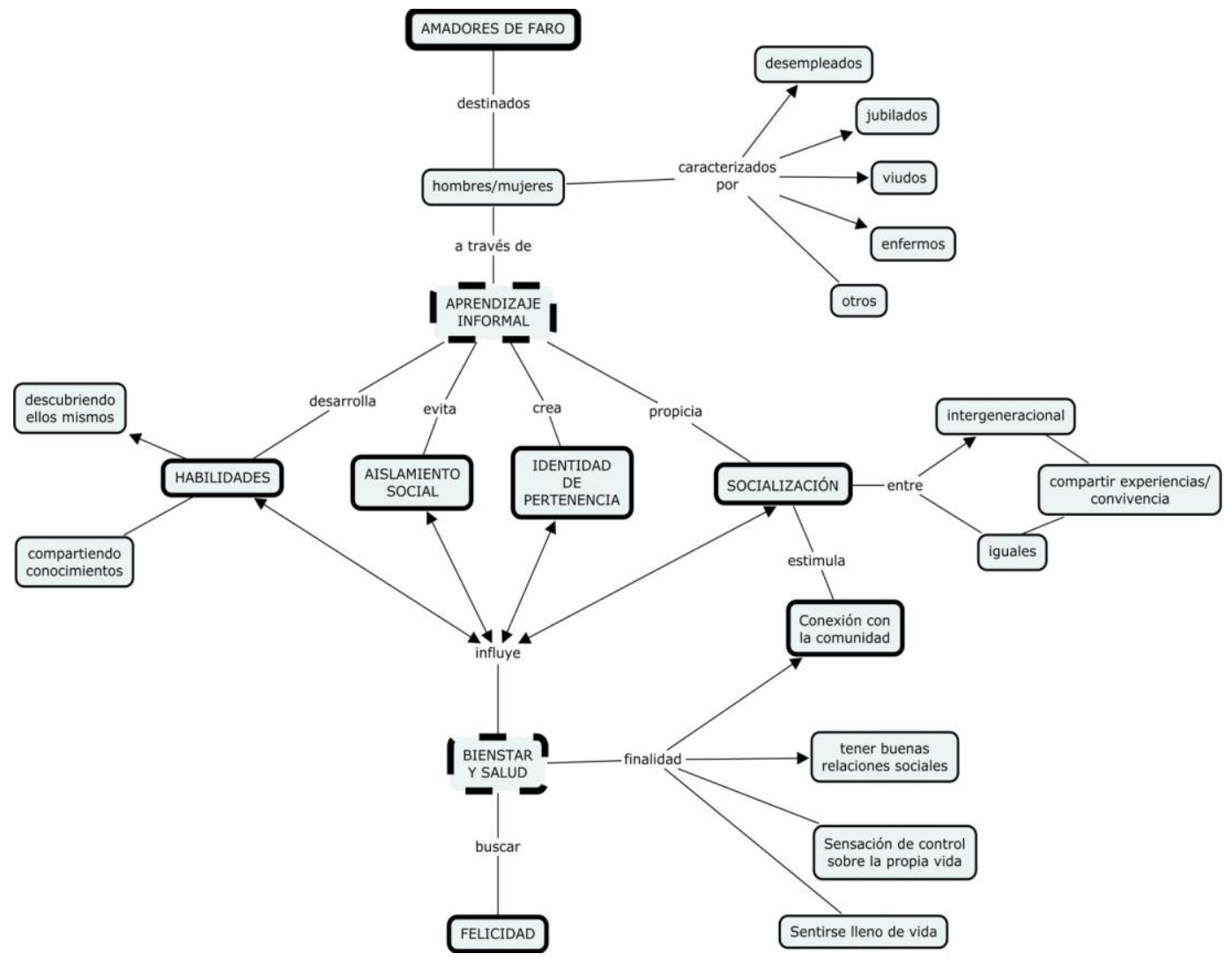

Figura 1. Mapa conceptual de las relaciones establecidas entre los conceptos objeto de estudio

El análisis se completó interpretando las diferentes unidades identificadas con el fin de conocer la percepción que tienen los mayores en cuanto a las implicaciones que tienen los Men's Shed en la mejora de la salud y el bienestar, el aprendizaje informal, la conexión con la comunidad, el sentido de pertenencia y la socialización. Se han identificado las repuestas más representativas en cada una de las categorías estudiadas por ser las más representativas en cuanto al universo colectivo de los entrevistados. 


\subsection{Aprendizaje Informal}

Dentro del club Los Amadores de Faro se produce una peculiar forma de aprendizaje informal, donde se combinan los conocimientos y habilidades que poseen sobre la pesca (actividad principal) con otros ligados más al entretenimiento y ocio, como el billar y otros juegos de mesa, actividad secundaria.

Así pues, compartiendo sus conocimientos o descubriendo nuevas técnicas de pesca, se va dando el aprendizaje informal al tiempo que interactúan.

Sí, algunas de las personas aprenden aquí, oyendo a uno, oyendo a otros... Lo hice así (...) lo hice asan (...) y la gente va teniendo conversaciones unos con otros de como las cosas funcionan. Y la otra gente va practicando en el momento que está pescando. La gente dice: ¡Ah!, pues funciona o no funciona. O vamos a cambiar para otra cosa. Andamos siempre cambiando para ver si funciona (...). ENTREVISTA 3-SOCIO Y COLABORADOR (APRINF 15).

La actividad aquí es más la pesca, la pesca deportiva, en campeonatos regionales, viene gente que va por todo el mundo. Son competiciones dentro del Algarve organizados por la Casa de Faro y ABRA, que es una asociación dentro del Algarve, que tiene participación a nivel nacional. ENTREVISTA 1-SOCIO DEL CLUB (APRINF 1).

\subsection{Socialización}

El hecho de que se produzca este tipo de aprendizaje les mantiene ocupados realizando actividades de su gusto, evitando el aislamiento social que muchos sufren, por diversas circunstancias. Así, este tipo de iniciativas que lleva a cabo el club son efectivas para luchar contra los problemas de la soledad: problema habitual en el colectivo de mayores.

Muchos viven solos, ya son viudos durante muchos años y ellos pasan aquí tiempo con otros, juegan, se divierten y se distraen un poco, pienso que eso es importante. ENTREVISTA 5- SOCIO Y COLABORADOR DEL CLUB (CONV 14).

Los ancianos, los jubilados... porque esas personas (...) para que esas personas estén sentados en los bancos de los jardines o estén en sus casa encerrados, yo pienso que tienen mucho que ganar viniendo aquí y pasando las tardes. ENTREVISTA 4-SOCIO Y PESCADOR PROFESIONAL (CONV 14).

Estos factores promueven el aprendizaje y se produce también una importante socialización entre los participantes. Se promueve las relaciones sociales entre iguales, conociendo personas y haciendo amistad, conviviendo día a día, compartiendo experiencias y creando un clima de compañerismo.

Me gusta bastante convivir con los socios, hablar con ellos, sean o no pescadores. Me gusta en general convivir aquí con las personas. ENTREVISTA 4- SOCIO Y PESCADOR PROFESIONAL (CONV 14).

Pienso que este club es importante para nosotros porque es un sitio donde nos podemos encontrar, conversar, convivir, jugar y participar en las actividades del club. ENTREVISTA 3- SOCIO Y COLABORADOR DEL CLUB (CONV 14).

Por otro lado, además de entre iguales, se da otro tipo de socialización bidireccional con jóvenes, donde se transmiten experiencias, conocimientos y habilidades sobre la pesca, produciéndose así una importante y beneficiosa relación intergeneracional.

Como resultado de esta relación intergeneracional, este club portugués se encuentra entre los tres mejores y con más trofeos regionales, nacionales y a nivel mundial. Aunque no cuenta con recursos económicos para tener entrenadores, ni formación profesional, son ellos mismos los que van pasando sus conocimientos de generación en generación de manera informal. 
Los principales conocimientos son aquellos que los más viejos nos enseñan, o sea, yo ya conocí a personas de otras generaciones los cuales me transmitían muchas cosas, las cuales yo hoy también acabo por transmitir a los más jóvenes (...) Yo pienso que se aprende mucho y nosotros intentamos transmitir eso a los más jóvenes o a las personas que después van a seguir nuestros pasos. ENTREVISTA 4- SOCIO Y PESCADOR PROFESIONAL (SOCINTER 16).

\subsection{Conexión con la comunidad}

La importancia de la labor del club radica en implicar no sólo a la comunidad de pescadores socios sino a todas las personas que quieran incorporarse y ser socios. Además, prestan ayuda a clubes similares a la hora de formar a otros mayores que quieran mejorar y amplían su radio de acción a través del voluntariado a otras personas necesitadas a través del banco de alimentos de la ciudad de Faro.

Cuando se hace un concurso de pesca, los peces cogidos son donados para la Casa [asociación que trabaja con personas sin hogar o personas adultas que no tienen recursos económicos]... exactamente llevaron 118 kilos de peces, por lo tanto ayudamos. ENTREVISTA 3- SOCIO Y COLABORADOR (COM 3).

También participan en labores de voluntariado medioambientales para la conservación del principal recurso económico de la ciudad -la Ría Formosa y la zona que la rodea.

El año pasado, el Ayuntamiento nos invitó a participar en la recogida de basura en las islas y nosotros ayudamos. Cuando hay algún tipo de evento relacionado con el mar o la ría, siempre estamos presentes. ENTREVISTA 4- SOCIO Y PESCADOR PROFESIONAL (COM 3).

\subsection{Sentimiento de pertenencia}

A través de todas las actuaciones puestas en marcha en el club los mayores sienten que pertenecen a algo concreto, su club, y para ellos esto es un hecho importante porque es como si se tratase de una gran familia. Este hecho se ve reflejado en los argumentos que se apuntan en las diferentes entrevistas hechas a los socios del club:

Y nosotros nos sentimos en casa, nos sentimos como una familia. ENTREVISTA 1- SOCIO DEL CLUB (SENTPERT 6).

Este es mi club y el de él, desde hace más de 20 años; por lo tanto, después de jubilarnos nosotros tenemos que ir a algún lado. Entonces nosotros vamos para nuestro club. ENTREVISTA 3-SOCIO Y COLABORADOR DEL CLUB (SENTPERT 6).

Estamos en nuestra casa, estamos a gusto aquí. ENTREVISTA 3- SOCIO Y COLABORADOR DEL CLUB (SENTPERT 6).

Sentir que son parte de algo hace a los participantes construir su propia identidad dentro de un grupo con el que pueden mantener relaciones enriquecedoras, evitando el aislamiento social propio de esta etapa de vida. Este fenómeno produce en ellos efectos positivos, pues como ellos mismos comentan, se sienten bien formando parte de esta gran familia.

\subsection{Bienestar y salud}

En conjunto, los factores especificados (desarrollar y transmitir nuevos conocimientos, lucha contra la soledad, socialización) asociados al aprendizaje informal producen una influencia positiva directa sobre la salud y el bienestar de los mayores.

Así pues, los mayores experimenten cambios en sus vidas, mejorándolas. A través de estas acciones los mayores se sienten llenos de vida, mejoran sus relaciones sociales, experimentan una sensación de 
control sobre sus vidas y entablan una conexión con la comunidad, acercándose a la felicidad.

Nosotros tenemos que hacer realmente ese trabajo que es traerlos para aquí y hacer que se sientan bien, o sea, hacer este trabajo es muy gratificante. Hasta yo considero estos trabajos de dirección muy útiles para la sociedad. En el caso de las personas más mayores, pienso que llevamos a cabo un trabajo muy saludable. ENTREVISTA 4- SOCIO Y PESCADOR PROFESIONAL (BIEN 35).

¿Qué cuáles son las ventajas de ser miembro de este club? (...) pues el simple hecho de estar aquí hace que las personas no entren en paranoia, que salgan de casa (...). ENTREVISTA 2- SOCIO DEL CLUB (BIEN 35).

\section{Discusión y conclusiones}

Debido a que cada vez la esperanza de vida es mayor, las comunidades basadas en programas de Men's Shed se han convertido en iniciativas útiles para promover y mejorar la salud psicosocial (Labonte,1997; Ballinger et al. 2009; Wilson y Cordier, 2013) y el bienestar en personas mayores a través de asociaciones comunitarias, en paises como Australia, Portugal e Irlanda.

El estado de bienestar en las sociedades avanzadas actuales incide en la importancia de la salud y el bienestar de nuestros mayores como indicador clave de la calidad de vida. Así pues, investigadores, políticos y profesionales de la salud estudian desde distintas ópticas los efectos de este tipo de comunidades de práctica y su indicencia en la salud y el bienestar. Este fenómeno se considera relativamente reciente y ha tenido un impacto social en sociedades anglosajonas en las dos últimas décadas.

Por ello, estos espacios pueden ser considerados como elementos clave para el diseño de políticas sociales que impliquen tanto la participación de hombres como de mujeres en contextos de aprendizaje informal. Ya que, según Golding (2011a, 2011b); y Golding et al. $(2007,2008)$ los Men's Shed ofrecen un contexto de aprendizaje informal que se adapta mejor a los hombres que los tradicionales centros de aprendizaje.

Así pues, se puede aceptar que el hecho de participar en este tipo de programas contribuye a mejorar la calidad de vida de los mayores en general, y específicamente de quienes se encuentran en riesgo de exclusión social. En este estudio los hombres participantes han encontrado sentido a la vida al participar de forma activa en su club, lo que les hace sentirse útiles y necesarios, y les sirve para reforzar su integridad, autoestima, sentido de pertenencia y bienestar personal.

Sin embargo, una de las debilidades de este proyecto es que no es generalizable debido a que sólo se entrevistó a miembros de un club de la región del Algarve. Sería necesario plantear futuras investigaciones en este sentido y seleccionar una muestra representativa de espacios comunitarios que promueven algún tipo de aprendizaje informal y analizar las implicaciones de los Men's Shed en la mejora de la salud y el bienestar de los mayores, el aprendizjae informal a través del voluntariado, la convivencia intergeneracional y la socialización de grupos, entre otras cuestiones.

Aunque la literatura científica revisada centra sus estudios en mayores sería interesante estudiar la posibilidad de transferir este tipo de iniciativas a organizaciones que trabajan con jóvenes en riesgo de exclusión social, fracaso escolar o conductas antisociales, entre otras.

\section{Agradecimientos}

La investigación desarrollada estuvo apoyada por el Proyecto Older Men as Active Learners (OMAL) dentro del Programa Europeo de Aprendizaje Permanente, Acción Grundtvig Learning Partnership. En él participaron socios de cuatro países europeos: Eslovenia (Andragoško društvo), Malta (Dingli Local Council), Estonia (Tallinna Ulikool) y Portugal (Universidade do Algarve). 


\section{Referencias}

Ballinger, M., Talbot, T. y Verrinder, K. (2009). More than a place to do woodwork: A case study of a community-based men's shed. Journal of Men's Health, 6(1), 20-27.

Creswell, J.W. (1998). Qualitative inquiry and research design. Thousand Oaks, CA: Sage.

Earle, L., Earle, T. y Von Mering, O. (1996). Sheds and male retirement. The place to go to... and come back from. Australasian Leisure Pleasure Journal, 1(1), 5-19.

Field, J. (2009). Well-being and happiness. Reino Unido: NIACE.

Fitzpatrick, T., Spiro, A., Kressin, N., Greene, E. y Bosse, R. (2001). Leisure activities, stress and health among bereaved and non-bereaved elderly men: the normative aging study. OMEGA, 43(1), 217-245.

Formosa, M. (2012). Education and older adults at the University of the Third Age. Educational Gerontology, 38(2), 114-126. doi:10.1080/03601277.2010.515910

Golding, B. (2011a). Social, local and situated: recent findings about the affectiveness of older men's informal learning in community contexts. Adult Education Quarterly, 61(2), 103-120.

doi:10.1177/0741713610380437.

Golding, B. (2011b). Shedding ideas about older men's learning. Lifelong Learning in Europe, 16(2), 119124.

Golding, B., Foley, A. y Brown, M. (2007). The international potential for men's shed-based learning. Journal for Continuing Liberal Adult Education, 34, 9-13.

Golding, B., Foley, A., Brown, M. y Harvey, J. (2009). Senior men's learning and wellbeing through community-based participation in Australia (Report for National Seniors Productive Ageing Centre). Victoria, Australia: University of Ballarat.

Golding, B., Kimberly, H., Foley, A. y Brown, M. (2008). Houses and sheds in Australia: an exploration of the genesis and growth of neighbourhood houses and men's sheds in community settings. Australian Journal of Adult Learning, 48(2), 237-262.

Jelenc-Krašovec, S. y Kump, S. (2009). Adult learning activities, social networks and different neighborhoods. European Societies, 11(2), 257-282.

Labonte, R. (1997). Power, participation and partnership for health promotion. Nueva Zelanda: Vic Health.

McGivney, V. (2004). Men earn, women learn: Bridging the gender divide in education and training. Reino Unido: National Institute of Adult Continuing Education.

Misan, G., Haren, M. y Ledo, V. (2008). Men's Sheds: a strategy to improve men's health. Nueva Gales del Sur: Mensheds Australia.

Patton, M. (2002). Qualitative research and evaluation methods. Thousand Oaks, CA: Sage.

Robertson, S., Galdas, P. M., Mccreary, D. R., Oliffe, J. y Tremblay, G. (2009). Men's health promotion in Canada: Current context and future direction. Health Education Journal, 68(4), 266-272. doi:10.1177/0017896909348785 
Smith, J.A., White, A.K., Richardson, N., Robertson, S. y Ward, M. (2009). The men's health policy contexts in Australia, the UK and Ireland: advancement or abandonment? Critical Public Health, 19(3 y 4), 427-440.

Stake, R. E. (2010). Investigación con estudio de casos. Madrid: Morata.

White, A. y Holmes, M. (2006). Patterns of morbidity across 44 countries among men and women aged 15-44. Journal of Mens Health and Gender, 3(2), 139-151.

Wilson, N. y Cordier, R. (2013). A narrative review of Men's Sheds literature: reducing social isolation and promoting Men's health and well-being. Health and Social Care in the Community, 21(5), 451-463. doi:10.1111/hsc.12019 